FORMATION Formation emploi

Revue française de sciences sociales

138 | Avril-Juin 2017

La professionnalisation dans l'enseignement supérieur : formes et effets variés

\title{
Entre universitarisation et « professionnalisation » : la formation des assistant-e-s de service social, en France et en Italie
}

Between academization and "professionalization" : the vocational training of social worker in France and Italy

Zwischen, Universitarisierung "und Professionalisierung: die Ausbildung von Sozialarbeitern in Frankreich und Italien

Entre universitarización y "profesionalización" : la formación de los/las

asistentes sociales en Francia e Italia

\section{Ruggero lori}

\section{OpenEdition \\ Journals}

Édition électronique

URL : http://journals.openedition.org/formationemploi/5071

DOI : 10.4000/formationemploi.5071

ISSN : 2107-0946

Éditeur

La Documentation française

Édition imprimée

Date de publication : 17 juillet 2017

Pagination : $39-58$

ISSN : 0759-6340

Référence électronique

Ruggero lori, «Entre universitarisation et « professionnalisation » : la formation des assistant-e-s de service social, en France et en Italie », Formation emploi [En ligne], 138 | Avril-Juin 2017, mis en ligne le 10 juillet 2019, consulté le 30 octobre 2020. URL : http://journals.openedition.org/formationemploi/ 5071 ; DOI : https://doi.org/10.4000/formationemploi.5071 


\title{
Entre universitarisation et «professionnalisation » : la formation des assistant-e-s de service social, en France et en Italie
}

\begin{abstract}
RUGGERO IORI
Doctorant en sociologie et ATER (Attaché temporaire d'enseignement et de recherche) à I'université de Versailles-Saint-Quentin-en-Yvelines, membre du laboratoire PRINTEMPS

(Professions, institutions, temporalités) UMR 8085 CNRS/UVSQ'.
\end{abstract}

Résumé

Entre universitarisation et « professionnalisation » : la formation des assistant-e-s de service social, en France et en Italie

Cet article interroge les transformations de l'enseignement supérieur sous l'effet des récentes réformes européennes, à la faveur notamment de l'introduction de la formation professionnelle dans l'espace universitaire. Partant du cas de la formation d'assistant-e de service social, il s'agit, d'une part, de questionner son rapport à l'université (en tant qu'espace et dispositif de formation spécifique) dans une perspective comparative historique et géographique (la France et l'Italie) et, d'autre part, de mettre en perspective les débats dans l'espace de l'enseignement supérieur des deux pays.

Mots clés : Assistant social, enseignement supérieur, professionnalisation de l'enseignement, politique de l'éducation, université, création-rénovation de diplôme, Union européenne, France, Italie, comparaison internationale

Abstract

Between academization and "professionalization": the vocational training of social worker in France and Italy

This article explores how European policies introducing higher vocational studies have transformed university systems in Italy and France. Our empirical analysis offers an overtime, cross-national comparison of professional training for social workers, investigating its role within the university as a specific (educational) field and as a training device. Our results thus offer crucial insights on competing understandings of higher education in the two countries.

1. Je tiens à remercier Aurélie Gonnet et Julie Minoc pour leurs relectures et conseils, ainsi que les rapporteurs de la revue et les coordinateurs du numéro pour leurs remarques. 
keywords: Social worker, higher education, professionalisation of teaching, education policy, university, diploma creation - diploma updating, European Union, France, Italy, international comparison

Journal of Economic Literature: I 23 I 28

Traduction : Auteur.

La réforme dite «LMD » (licence-master-doctorat) en France, ou « 3 + 2 », en Italie, a été introduite au début des années 2000 dans la plupart des pays européens, suite à la déclaration de Bologne. Elle a inscrit formellement la formation professionnelle au sein de l'enseignement supérieur et a conduit, dans le même temps, à une standardisation des cursus, à l'image de la "formation permanente ». Au principe de cette réforme se trouve une conception renouvelée de l'individu comme acteur rationnel qui construit son parcours professionnel individualisé en choisissant des "modules" (Tanguy, 2005). L'idée de "formation tout au long de la vie ", introduite par les traités européens (protocole de Lisbonne), témoigne aussi de ce mouvement d'ensemble. Au sein des formations de "travail social " ${ }^{2}$, espace à la frontière entre enseignement supérieur et formation professionnelle pour adultes, entre formation initiale et continue, donnant accès à des titres "protégés ", le processus de Bologne a été accueilli avec enthousiasme. Cette réforme répond tant à une volonté de revalorisation de ces formations, via l'universitarisation, qu’à une logique de " professionnalisation " propre à ce secteur.

Pour autant, au-delà du modèle théorique, l'homogénéisation européenne en la matière est encore loin d'être aboutie. Les contours des filières du travail social connaissent d'importantes disparités en Europe, selon les spécificités des systèmes d'enseignement nationaux. Certaines sont pleinement inscrites dans le système universitaire, d'autres en sont autonomes; certaines s'établissent dans des hautes écoles professionnelles ou encore sont reconnues selon leur niveau de qualification professionnelle. En France, bien qu'une reconnaissance de 180 ECTS (crédits européens, de niveau $\mathrm{Bac}+3$ ) ait été introduite, ces formations restent aujourd'hui au niveau III de qualification professionnelle $(\mathrm{Bac}+2)$, alors que des discussions sur l'entrée à l'université, l'autonomie dans des Hautes Écoles Spécialisées, ou encore la mise en place d'un tronc commun entre plusieurs métiers, font l'actualité du champ depuis plusieurs années (Jaeger, 2012 ; Molina, 2014). Depuis les années 1960, se pose la question du rattachement universitaire de ces formations, mais la plupart des acteurs du champ revendiquent leur éloignement du système académique et la spécificité de savoirs "professionnels »

2. L'espace du " travail social " regroupe, dans le sens commun, des activités et des formations diverses, loin d'être clairement définies. Pour des raisons de lisibilité, tout au long du texte, nous utiliserons ce terme sans les guillemets. 
(Rullac, 2014) $)^{3}$ face aux disciplines universitaires. En Italie, au contraire, à l'instar d'autres formations professionnelles de l'éducation et de la santé, l'adoption des directives européennes a conduit à l'entrée de la filière de "service social " dans l'institution universitaire. Or, comment expliquer qu'une même réforme ait pu se matérialiser si différemment ? En quoi les spécificités des systèmes d'enseignement nationaux jouentils dans les politiques à l'œuvre dans cet espace ? La comparaison de ces deux cas de figure vise à mettre en perspective les débats en cours dans l'espace français pour comprendre les reconfigurations à l'œuvre dans ce champ. En resituant le contexte des changements socio-historiques concomitants aux réformes législatives, les stratégies opérées dans chacun des pays interrogent la distinction formation/enseignement dans le cadre des politiques de "professionnalisation " de l'enseignement supérieur, plus spécifiquement dans une filière féminine et "moyenne ", comme celle qui mène au titre d'assistant-e de service social.

Dans la première partie, nous montrerons, à partir du cas italien, comment l'universitarisation du cursus en service social participe conjointement à une progressive professionnalisation du corps enseignant et à l'introduction de logiques professionnelles dans la sphère universitaire. Dans une seconde partie, nous partirons de l'exemple français pour illustrer comment la tentative de rapprochement vis-à-vis du modèle académique ne conduit pas ici à l'entrée de cet espace de formation dans l'université, les statuts des enseignants demeurant plus hétérogènes et le lien avec le marché de l'emploi plus fort.

\section{Encadré 1 : Méthodologie}

Cet article s'appuie sur une recherche doctorale en cours traitant de la formation en service social, en Italie et en France. II repose principalement sur une analyse socio-historique de cet espace, réalisée sur la base d'un traitement primaire et secondaire de différentes sources écrites : archives des formations, publications dans des revues et articles de presse spécialisée, lois, arrêts et rapports de l'administration de l'action sociale dans les deux pays, entretiens.

En complément, nous avons mobilisé ici des données issues d'une enquête de terrain à la fois qualitative et quantitative, menée entre 2012 et 2016, dans quatre instituts de formation dans les deux pays, deux en région parisienne et deux dans une ville du nord de l'Italie.

L'articulation de plusieurs critères a présidé à ce choix : les conditions d'accès au terrain dans les deux pays, la place de ces instituts dans l'histoire institutionnelle du champ, le nombre d'étudiants inscrits (pour permettre la comparaison quantitative) et la nature de leur tutelle (privée ou publique).

3. En dehors, entre autres, du groupe RIFF (Réseau interuniversitaire de formation des formateurs) aujourd'hui RUFS (Réseau universitaire des formations sociales). 
Outre une enquête longitudinale par suivi de cohorte (533 étudiants dont 280 suivis quantitativement, 25 qualitativement ; enquête FRITA 2013-2016), nous avons mené des observations tout au long des trois ans du cursus, lors de différents moments de la formation : entretiens de sélection, réunions de pré-rentrées, cours et séminaires, réunions pédagogiques, etc. Ces observations ont été complétées par des entretiens formels et informels avec différents acteurs de la formation : du formateur responsable pédagogique aux enseignants, des vacataires aux "personnalités » historiques du champ. Vingt-neuf entretiens ont été conduits et enregistrés dans ce cadre (16 en Italie, 13 en France) autour de l'histoire de la formation, des pratiques pédagogiques et professionnelles, des rapports avec l'Université, ou encore des trajectoires individuelles de ces personnes. Les extraits mobilisés dans l'article font partie de ce corpus et ont été traduits par l'auteur.

Enfin, parallèlement à cette enquête de terrain dans les quatre instituts, des observations ont également été effectuées sur la même période à l'occasion d'événements importants, à l'échelle nationale et internationale, autour de la "recherche en travail social » : des séminaires de recherche, des journées d'études, des colloques internationaux. À cet égard, notons que cet espace de formation a été abondamment étudié et a donné lieu à une littérature foisonnante aussi bien dans des revues scientifiques, que dans des revues professionnelles ou des ouvrages spécialisés. Cela a constitué un matériau à part entière, en particulier les articles et les ouvrages portant plus spécifiquement sur la formation en tant que telle et les rapports avec les pouvoirs publics. Une attention particulière a été accordée au statut du rédacteur. L'étude scrupuleuse des revues spécialisées (Vie Sociale, Revue Française de Service Social, Rivista di servizio sociale, Rassegna di Servizio Sociale) et des publications liées aux Associations nationales des assistants sociaux - ANAS -, spécifiques à chacun des deux pays a notamment permis de repérer et mettre en perspective les différentes prises de position au fil des années, ainsi que d'identifier l'organisation du champ.

Il pourrait sembler paradoxal qu'un pays comme l'Italie, où s'observent de nombreuses inégalités, à la fois territoriales depuis son unification, socio-économiques ou en termes d'emploi eu égard au poids notamment de l'économie informelle, ait tardé à institutionnaliser le travail social. Ce n'est en effet qu'après la Seconde Guerre mondiale qu'une forme d'État social italien se structure conjointement à la constitution de la République, sur les cendres de l'ancien Royaume d'Italie et après vingt ans de régime fasciste. Sous l'impulsion du patronat, des élites intellectuelles et politiques et des financements étrangers (notamment américains via le Plan Marshall) prolifèrent, jusqu’aux années 1970, des dizaines d'écoles " sociales ", inégalement distribuées sur l'espace national. Ces écoles, formant principalement au métier d'assistant-e social-e, suivent des approches idéologiques diverses (confessionnelles ou laïques, avant tout d'inspiration catholique et libéral-socialiste) et tentent, à plusieurs reprises (1953, 1958, 1961, 1963, 1975, 1977, 1979, 1981) d'obtenir une reconnaissance légale de la part des pouvoirs publics, sans succès.

À la différence de l'Italie, l'État social français se structure tout au long du XIXe siècle, et notamment lors de la Troisième République. Formations originellement professionnelles, les premières écoles de travail social naissent au début du XXe siècle, au croisement du sanitaire et du social (Rater-Garcette, 1996). Le métier d'assistant-e de service social 
s'institutionnalise dès la fin des années $1930^{4}$, pour prendre sa forme actuelle après la Libération. Tout au long des années 1960 et 1970, d'autres métiers se développent, le bassin de recrutement s'élargit, d'autres formations apparaissent (notamment celle d'éducateur), et une partie de la formation professionnelle est prise en charge par l'université (suite à la création des IUT - Institut universitaire de technologie - et à la participation des universités à la formation professionnelle continue ${ }^{5}$ ) qui commence à concurrencer ces filières. La formation aux métiers du social reste néanmoins privée, sous tutelle du ministère de la Santé et des Affaires sociales, à la différence de la grande majorité des formations chapeautées elles par l'Éducation nationale ou le ministère de l'Enseignement supérieur et de la Recherche (ESR). Si ces filières ne sont pas les seules à être séparées de l'institution universitaire, comme par exemple les STS (sections de techniciens supérieurs) ou les classes préparatoires, les liens avec celle-ci demeurent conflictuels, critiquée qu'elle est pour son académisme et son éloignement du « terrain ».

C'est au cours des années 1980 que la formation au "social " change de forme dans les deux pays. La création des régions administratives, à la fin des années 1970, et le processus de décentralisation produisent un transfert des compétences, tant du côté de la formation professionnelle que de celui des politiques sociales transversales, qui contribue à réorganiser ces cursus. Les contacts entre les acteurs de la formation en service social et le champ universitaire se font alors de plus en plus concrets, bien qu'ils prennent forme dans deux configurations de formation distinctes dans chacun des deux espaces nationaux.

\section{De la «formation » à l' « enseignement »}

Au début des années 1980, le territoire national italien compte 109 écoles qui offrent une formation d'assistant-e social-e, en plus de sept écoles de statut universitaire agréées. Ces écoles proposent un cursus en trois ans, alternant cours et stages, les premiers étant dispensés par des enseignants du secondaire, des enseignants universitaires ou des assistantes sociales. Plusieurs tentatives de réformes ont été amorcées pour leur reconnaissance, sans succès, notamment à cause de la fermeture et l'intermittence du système politique partisan de l'époque, qui compliquait les discussions parlementaires sur le long terme. La méfiance de cet espace professionnel envers le secteur public, considéré comme incapable de transmettre les "valeurs » spécifiques du service social, demeure forte, tout comme le désir d'autonomie sur la formation pour en garder le caractère " expérimental ». La volonté

4. Si les premières écoles françaises sont fondées dès le début du XXe siècle, le premier diplôme d'État commun aux infirmières date de 1938.

5. Circulaire du 26 avril 1972. 
d'une reconnaissance légitime est patente et transparaît dans les échanges internes à cet espace qui se tiennent à l'époque (Bernocchi et alii, 1984, p. 310).

Pourtant, les débats de ces années oscillent entre l'intégration au système de formation professionnelle des régions et la reconnaissance universitaire. Certaines écoles avaient déjà commencé à organiser la formation dans le cadre des centres universitaires agréés (Scuola diretta a fini speciali, trad. litt. École dirigée à des fins spéciales $\left.{ }^{6}\right)$, sorte de structure intermédiaire encadrant plusieurs diplômes technico-professionnels, alors que d'autres écoles avaient été incorporées à l'organisation des collectivités locales (notamment communales ${ }^{7}$ ). En 1982, ${ }^{8}$ le législateur réorganise ces écoles " spéciales " en intégrant progressivement la formation initiale professionnelle au système universitaire. En 1985 et $1987^{9}$, deux décrets fondateurs inscrivent plus spécifiquement la formation au service social au sein de l'université, avec un programme pédagogique défini par décret ministériel. Tel un "champ organisationnel ", des processus d' "isomorphisme " ${ }^{10}$ (Di Maggio et Powell, 1983) contribuent à homogénéiser progressivement cet espace. Dès lors, des écoles ferment ou fusionnent, créant des centres universitaires agréés ou rejoignant des universités existantes. Cela contribue en partie à déposséder les responsables de formation de l'époque de leur marge de manœuvre sur les cursus de formation ${ }^{11}$. Le programme des études n'est plus organisé singulièrement par les écoles, mais commence à être géré par les universités selon les plans ministériels. Il en va de même pour la sélection du personnel enseignant, parmi lesquels se trouvent les professeurs « en poste » qui encadrent de droit les formations.

Les années 1990 se caractérisent par une période de reconfiguration de la gestion de l'enseignement supérieur et du social, dépendant en grande partie d'une recomposition des rap-

6. Ces écoles, rattachées à l'Université et instituées par une loi de 1933, étaient à l'époque au nombre de huit (par ordre de création) : Sienne, Rome, Florence, Parme, Pise, Pérouse, Rome-Magistère de Maria Santissima Assunta (aujourd'hui LUMSA), et Cagliari.

7. Pour prendre le cas d'une ville du Nord de l'Italie, cette école fusionne les traditions des deux écoles existant auparavant (qui renvoyaient à la tradition catholique et laïque) dans une école communale centrale. Les formateurs salariés de cette école sont alors intégrés à la fonction publique territoriale et les diplômés à la sortie peuvent être embauchés dans les services publics locaux. Ce rattachement a cours de 1978 aux années 1990, quand l'école est transformée progressivement en centre universitaire agréé et prendra part à la création de la nouvelle université publique de la ville à la fin des années 1990 .

8. Décret présidentiel n 162 du 10/03/1982.

9. Décret ministériel n 35 du 30/04/1985 et Décret présidentiel n. 14 du 15/01/1987.

10. Dans leur analyse du champ organisationnel et des phénomènes de bureaucratisation, Di Maggio et Powell utilisent le concept d'isomorphisme pour indiquer les processus qui amènent les organisations à homogénéiser leurs normes. Cet "isomorphisme instutionnel " peut prendre trois formes : mimétique, normatif ou coercitif.

11. Comme nous le révèle un ancien formateur à propos de l'école évoquée supra : "et ensuite est arrivée l'université... Il y a eu toute une phase de cogestion - on peut l'appeler comme ça, et c'est là que les anciennes assistantes sociales historiques se sont rendues compte, selon moi, qu'elles perdaient quelque chose... qu'elles se sont fait vraiment piquer le pognon... ». Entretien avec Franco, 70 ans, pendant longtemps formateur pour l'école communale et ensuite collaborateur indépendant pour diverses formations du social jusqu'aux années 2000. 
ports de force au sein de l'univers politique partisan (Mastropaolo, 2000). La réforme dite "Ruberti ", en 1990, ${ }^{12}$ initie le mouvement d'autonomisation des universités et intègre les prémices du financement privé (Pitzalis, 2007). L'Université s'autonomise progressivement, pédagogiquement et financièrement. Le fonctionnement universitaire commence alors à se généraliser pour les cursus de service social, la création du diplôme universitaire (DU, cycle court professionnel) de service social, en 1993, participe de l'institutionnalisation de ce cursus. Sous le modèle des professions libérales, l'obtention du DU, le passage par un concours national (it. Esame di Stato) et l'inscription à un ordre professionnel créé dans la même année ${ }^{13}$, permettent l'entrée " officielle " dans le métier.

Les évolutions de cette formation doivent se lire alors à l'aune des changements qui traversent l'institution universitaire. La signature de la déclaration de Bologne, en 1999, amène à l'introduction soudaine de principes européens au sein de l'institution universitaire. Les cycles universitaires " classiques » de la Laurea (correspondant au niveau " mầtrise ", de 4 à 6 ans) voient leur durée réduite à 3 ans et sont uniformisés avec les DU professionnels au niveau licence. Cette réforme est alors promue comme une manière de lutter contre les très faibles taux de réussite à l'université (40\%) et la durée longue des études, cette dernière engendrant une sortie tardive du système de formation (60\% terminaient leurs études à 26,7 ans) (Woolf, 2003). De la même manière, la "modernisation » de l'institution universitaire permet d'attirer de nouvelles masses d'étudiants, la "massification " du public universitaire n'ayant pas eu vraiment lieu par rapport à d'autres pays ${ }^{14}$.

Le service social est alors officiellement intégré dans des départements universitaires et, selon les contextes locaux, au sein des filières de sociologie, science politique, droit ou encore dans des cursus interdisciplinaires de diverses universités. Le mécanisme de légitimation qui se dessine ici passe alors par un processus de " poussée académique » observé aussi dans d'autres systèmes d'enseignement européens (" academic drift»; Neave, 1979), les écoles professionnelles adoptant progressivement le modèle universitaire. Non seulement la formation est désormais " créditée " de 180 ECTS, mais le passage du DU à la licence est presque immédiat. En 2015-2016, on compte 40 licences en "sciences du service social $"{ }^{15}$ et 38 masters, ainsi qu'une dizaine de parcours doctoraux spécifiques. Les maquettes et les cursus sont directement gérés par le ministère de l'Université, mais l'offre de formation permet à chaque structure d'y apporter ses spécificités, nourrissant la concurrence entre universités et la sélection des étudiants. La validation des trois ans permet d'obtenir une licence, suite à laquelle chaque étudiant peut librement choisir de

12. Loi n 341 du 19/10/1990, par le nom du professeur Antonio Ruberti, ministre de l'Université et de la Recherche sous le gouvernement démocrate-chrétien, ancien doyen de l'université de Rome.

13. Décret ministériel $n^{\circ} 180$ du 23/07/1993, et loi nº 84 du 23/03/1993, création du DUSS (diplôme universitaire supérieur spécialisé) et de l'ordre professionnel.

14. Selon les données de l'ISTAT, Institut national italien de statistique, en 2013, en Italie, $23 \%$ des 30-34 ans suivent un cursus post-bac, contre $44,1 \%$ des français pour la même classe d'âge.

15. Le label « science » s'impose dans la plupart des filières du supérieur. 
rentrer dans le métier en s'inscrivant au concours, organisé conjointement par l'université et l'ordre professionnel régional, poursuivre en master, ou suivre ces deux voies en parallèle. Cette formation, largement féminisée, permet alors de rénover l'« image » de certaines filières en crise de recrutement, notamment la sociologie, attirant de " nouveaux " étudiants - ou plutôt de nouvelles étudiantes - et de nouvelles ressources dans une institution autonome financièrement ${ }^{16}$. De plus l'intégration dans l'espace universitaire contribue à asseoir la légitimité du groupe professionnel.

Cette progressive universitarisation entraîne tout d'abord la professionnalisation de son corps enseignant : désormais, comme toute autre discipline universitaire, des étudiants assistant-e-s de service social peuvent continuer leurs études en poursuivant dans la carrière académique. Dans l'une des deux universités observées (filière privée à orientation catholique), le cursus de service social est intégré au sein du département de sciences politiques et sociales, mais est organisé de manière autonome par un groupe d'enseignant-e-s chercheurs-ses qui ont développé leur propre orientation théorique, les approches scientifiques étant loin de faire consensus dans la communauté académique. Plus qu'un "academic drift ", ce cas traduit un véritable " research drift ".

Toutefois, en dépit de l'inscription universitaire, l'enseignement n'est pas assuré uniquement par des enseignants-chercheurs en service social. En réalité, les titulaires de cette discipline sont encore peu nombreux et dans la plupart des cas, ce sont des enseignants-chercheurs en sociologie, psychologie, droit ou science politique qui assurent les enseignements dans ces cursus. La pénurie des postes à l'université ne permet pas d'embaucher autant d'enseignants et cet espace est loin de représenter un " monde pacifié " de l'enseignement supérieur et de la recherche. Des enseignants vacataires, parfois doctorant-e-s en service social, ayant un titre d'assistant-e-s de service social, dispensent les modules plus "professionnels ", sur une thématique particulière. Pour ceux-ci, l'enseignement universitaire ne fait pas forcément consensus, et certains regrettent l'espace régional des écoles où la "vocation " des étudiants était selon eux plus prégnante et leur marge de manœuvre plus grande.

Comme nous le confie une enseignante vacataire d'une des deux filières (université publique), dans laquelle elle développe un cours original sur la "narration d'expériences du social »:

"... pour être synthétique et brutale : le cursus d'avant était une formation, celui-ci c'est de l'enseignement... Cela change tout, absolument tout, la formation est un processus qui accompagne, stimule et implique, interroge et bouge, dérange les personnes, et les aide à construire dans le temps une identité corrélée à cette spécificité professionnelle... et je ne m'exprime pas au hasard sur ces termes, parce que cette question vaut pour l'assistante

16. En guise d'exemple, selon les données de 2013 de l'OCDE (Organisation de coopération et développement économiques), les frais d'inscription représentent $20 \%$ du budget des universités italiennes, contre $64 \%$ aux États-Unis, $28 \%$ au Royaume-Uni, et $13 \%$ en France. 
sociale comme pour d'autres figures professionnelles. L'enseignement, c'est tout autre chose... (...). C'est pour cela que je parle de formation et d'enseignement. Le cours est absolument nécessaire, de psychologie générale, du développement, de sociologie de la déviance... et c'est autre chose que d'être face à des situations concrètes sur lesquelles on tombe dans la profession... et surtout être pris dedans... c'est pour cela que je trouve particulièrement critique le passage de la formation d'antan à celle universitaire... " ${ }^{17}$

Si l'entrée dans l'université demeure contestée par certains, les professionnels « du terrain " ne sont pas pour autant exclus de ces formations. L'introduction de ces filières professionnelles au sein de l'université a rendu de plus en plus étroit le rapport entre enseignement universitaire et marché de l'emploi. L'organisation pédagogique du service social (stages et alternance, modules spécialisés, interdisciplinarité) fait écho aux principes de la réforme de Bologne, qui met l'accent sur l'individualisation du parcours et sur le lien entre marché du travail et cursus universitaires.

Dans l'une des deux formations observées (université publique), la filière de service social est intégrée au département de sociologie, qui propose quatre cursus en licence (sociologie, service social, sciences du tourisme, et sciences de l'organisation). Le cursus de service social est, selon les mots de la responsable de la filière ${ }^{18}$, celui qui " fait $d u$ chiffre " et "n'a pas de competitors (concurrents) ". En matière d"insertion professionnelle, ce cursus obtient ainsi les meilleurs résultats selon les évaluations du ministère et de la présidence de l'université, ce qui est vecteur de meilleures ressources financières pour le département. Le lien et la connaissance du marché de l'emploi sont alors fondamentaux pour cette professeure qui met l'accent sur les "séminaires " (laboratori) dispensés. Dans ce cadre, des vacataires extérieurs, souvent responsables de structures sur le territoire, interviennent tout au long des trois ans. Elle-même, lors de son cours de "politique sociale » en deuxième année, décide d'inviter, à plusieurs reprises, des cadres du secteur associatif, pour qu'ils témoignent de la " réalité » du métier.

Un autre élément joue en faveur d'une transformation de la formation en service social sous l'effet du marché de l'emploi. En effet, d'après les chiffres nationaux des diplômés, la réduction des embauches dans le public amène une partie des assistants sociaux à se tourner vers le tiers secteur, à accepter des contrats à durée déterminée ou précaires, voire à quitter l'espace professionnel ${ }^{19}$ (May et Cacioppo, 2015, pp. 53-78). Pour

17. Entretien avec Laura, quinquagénaire, assistante sociale diplômée, exerçant en profession libérale tout en étant formatrice pour adultes dans des cursus socio-sanitaires (formation initiale et continue).

18. Discussion informelle avec Ada, sexagénaire, professeure associée (maître de conférences habilitée aux fonctions de professeur d'université) en sociologie, responsable de la filière. Elle a commencé sa scolarité dans une école de service social où elle a ensuite donné des cours en tant que formatrice. Après un diplôme en sciences politiques, elle a poursuivi sa carrière à l'université où elle est entrée en tant que maître-assistante de l'ancien doyen de la faculté de sociologie.

19. Dans une recherche collective menée sur les assistants sociaux en Italie, les chercheurs attestent que seulement $14 \%$ des nouvelles embauches sont en CDI (contrat à durée indéterminée), 27 \% en CDD (contrat 
contrer la réduction des emplois dans le public et l'affaiblissement des garanties professionnelles, les universités de la région, conscientes en partie du problème, tentent d'y remédier en développant un ensemble de dispositifs de formation qui entraînent une modification progressive du rôle de l'assistant-e social-e à la faveur d'une plus grande flexibilité et adaptabilité ${ }^{20}$.

Désormais, sous l'effet conjugué de l'injonction à se professionnaliser dès le secondaire et le changement des modalités de sélection des candidats (autrefois déléguées aux écoles), c'est le public même de ces cursus qui change. Plusieurs étudiants s'orientent directement vers ces formations en sortant de la maturità, le baccalauréat italien (16\% seulement des inscrits au niveau national ont plus de 23 ans, $8 \%$ dans notre échantillon, selon les données du ministère de l'Université italienne en 2016 et notre enquête FRITA). C'est donc à la frontière entre "professionnalisation » de l'université et universitarisation du service social que ce processus peut être saisi.

Le temps universitaire n'est donc plus un temps à part échappant aux impératifs sociaux, politiques ou économiques, mais un temps ancré dans le marché du travail. Alors que le rattachement universitaire pouvait laisser croire à un virage plus " académique " de ces cursus, c'est l'académie même qui change dans sa substance. Dans un mouvement de « rattrapage " des compétences à acquérir, l'accent est mis, d'un côté, sur la " professionnalisation ", de l'autre, sur l'orientation vers des emplois disponibles, qui ne correspondent que partiellement aux canons du métier. Les connaissances dispensées sont rarement remises en cause par les étudiants ou dans les échanges avec l'enseignante, et se concentrent pour souligner l'" efficience " du service social. Les cours deviennent alors une manière de parfaire le lien entre université et travail, constitué par une mission " publique " exercée de plus en plus avec les moyens du "privé », de s'adapter au réel d'un marché de l'emploi davantage précarisé.

à durée déterminée), 38,8 \% avec des collaborations et $20 \%$ sous contrats à projet ou atypiques. De même, seulement un tiers des diplômés interrogés exerce effectivement un travail d'assistant-e social-e (Tognetti, 2015).

20. Des passerelles entre le service civique ou le bénévolat et les études universitaires sont envisagées pour permettre un meilleur ancrage des diplômés à la sortie de la formation. Dans les cours en université, l'accent sur la polyvalence amène, par exemple, à un détournement de la signification du dispositif, allant souligner une polyvalence professionnelle qui s'apparente à une plus forte précarité de l'emploi, ce qui contribue à faire évoluer la licence en service social au-delà d'une formation à un "métier " spécifique et avec un caractère professionnel plus "généraliste". 


\section{Revendiquer l'écart pédagogique et institutionnel ?}

En France, comme en Italie, les années 1980 sont un moment de basculement entre ce qui va devenir la formation et le métier d'assistant-e social-e.

Tout d'abord, la réforme de $1980^{21}$ poursuit le rapprochement avec le modèle universitaire initié dès les années $1960^{22}$ (Morand-Durocher, 1992). Elle est l'aboutissement d'une série de négociations entre acteurs du travail social et pouvoirs publics, amorcées suite à un projet du ministère visant à abaisser les titres nécessaires à l'entrée dans ces formations ; en témoigne une ancienne directrice d'un centre de formation ${ }^{23}$ :

"... alors évidemment tout ça [le projet du ministère, nda] a mis beaucoup en émoi tous les responsables de centres de formations, les formateurs, les étudiants, tout le monde n'était pas content... Et donc y a eu un groupe de travail de négociation avec les syndicats, les représentants des associations, des employeurs, les professionnels... et y a eu une négociation très difficile (...) on a été obligé de tout réexpliquer depuis le commencement jusqu'à ce qu'était devenue la formation professionnelle maintenant. D'autant plus qu'on a créé le DSTS 24 avec le même directeur de l'action sociale, Guy Raffi, qui est devenu après administrateur de Montronge [aujourd'hui institut régional de travail social de Montrouge]... bon. Alors, cela a été très houleux, très compliqué, mais on a quand même réussi à faire une réforme qui a été plutôt une bonne reforme d'après ce qu'on nous a toujours dit... ".

La réforme de 1980 aboutit à la reconnaissance officielle de ces formations comme cursus post-bac, et conduit à intégrer des «théories du service social " pour un tiers du programme et à augmenter les heures des disciplines enseignées (sociologie, psychologie, droit), transformées en " unités de formation ». Un " mémoire de recherche » de fin d'études vient enfin consacrer la formation. Le stage représente un volume important sur les trois ans (quatorze mois) et le stage en polyvalence de secteur (cinq mois) est rendu obligatoire. Le poids de la conception " publique " du métier est patent ; pourtant, cette tentative de reconfiguration n'aboutit pas à une universitarisation de ce cursus. Ce qui demeure aussi particulier au cas français, c'est que ce travail social, censé exercer une mission d'intérêt général et d'utilité publique, n'est pas un "service public » en tant que tel. Si l'État délègue ces missions aux centres de formations et aux associations, les écoles continuent à former leurs étudiants selon diverses méthodes et

21. Décret $n^{\circ} 80-334$ du 6/05/1980 relatif à la formation des assistants de service social

22. Réforme de 1961 et arrêté du 2/04/1970.

23. Entretien avec Monique, sexagénaire, diplômée assistante sociale dans les années 50, elle poursuit des formations aux méthodes anglo-saxons et elle devient formatrice puis directrice d'école, avril 2013.

24. Le Diplôme Supérieur de Travail Social (DSTS, aujourd'hui Diplôme d'Etat d'Ingénierie Sociale, DEIS) est créé par arrêt du 14/10/1978 du ministère de la Santé et de la Famille. Il vise à tisser un premier lien avec l'université pour permettre aux travailleurs sociaux d'obtenir des maîtrises et des diplômes de l'enseignement supérieur. 
approches idéologiques. Ainsi, n’émerge aucune théorie originale du service social, ni des méthodes partagées; les liens entre différentes spécialités du travail social restent sporadiques et les savoirs inégalement transmis d'une école à l'autre (Viguier, 2010, p. 323).

Cette réforme s'inscrit de plus dans une mouvance particulière de la Direction de l'action sociale. Celle-ci se fonde sur la volonté, tant de la part des cadres du service social, formés dans l'après-guerre aux formations et aux méthodes anglo-saxonnes ${ }^{25}$, que de ceux de l'action sociale, d'améliorer la cohérence entre les formations et de conserver l'indépendance pédagogique et professionnelle des écoles face à l'Éducation nationale. Ces années se caractérisent par une génération particulière de hauts fonctionnaires, à la frontière entre monde politico-administratif et monde social, liés socialement aux cadres des écoles de l'époque. Guy Raffi, sous-directeur des professions sociales entre 1975 et 1983, s'inscrit dans ce " microcosme " qu'est la Direction de l'action sociale des années 1980 (Genieys, 1999), dans le sillage du "lénoirisme " ${ }^{26}$ et de cette vision technocratique de l'action sociale d'origine catholique ${ }^{27}$.

Avec le "tournant de la rigueur" des années 1980 (Bezes, 2009²), ces hauts fonctionnaires s'éloignent progressivement de l'idée - qui suivait le compromis keynésien de la " troisième voie " avec la Libération - d'un service public d'action sociale. Ils se réorientent vers le secteur associatif, proche socialement (notamment grâce aux socialisations catholiques et aux trajectoires sociales similaires) et intéressant financièrement (moins coûteux eu égard à la réduction budgétaire des subventions sociales) (Viguier, 2010). Une des conséquences de cette réorientation avait été notamment la démission, en 1982, de la ministre de la Solidarité nationale (ensuite Affaires sociales), Nicole Questiaux, proche de cette mouvance ${ }^{29}$. Dans une circulaire de la même année ${ }^{30}$

25. La pratique anglo-saxonne de travail social se structure principalement sur trois méthodes : le case work (individuel), le group work (collectif) et le community work (communautaire), importées en France initialement dans l'entre-deux guerres et ensuite à la Libération dans le cadre de moments spécifiques de formation. 26. Par René Lenoir, auteur de l'ouvrage, largement relayé à l'époque, "Les exclus ». Cet énarque, directeur général au ministère des Affaires sociales sous la présidence de Giscard d'Estaing (1974-1978), sera ensuite dans la Commission des affaires sociales du VIe Plan, directeur de l'ENA (Ecole nationale d'administration), et président de UNIOPSS (Union nationale interfédérale des organismes privés, sanitaires et sociaux à but non lucratif).

27. Pour une analyse plus détaillée de ce champ, voir l'important travail de thèse de Frédéric Viguier, en particulier la deuxième partie (Viguier, 2010).

28. P. Bezes met en valeur comment ce "tournant néo-managérial " marque la victoire des agents de l'État aux compétences transversales (notamment l'importance accrue du ministère des Finances) sur les agents sectoriels, avant que les nouvelles règles internationales et européennes rentrent au sein de l'État (Bezes, 2009). 29. Elle était notamment proche de Pierre Laroque, désigné comme le "père " de la sécurité sociale. Pour prendre connaissance du profil de cet énarque, voir Viguier, 2010, pp. 289-294.

30. Ministre réputée dans le milieu du travail social, dont la circulaire est régulièrement citée comme un temps fort dans la construction de cet espace. À ce propos, un dossier spécial " 30 ans après » lui est dédié, en 2012, par Vie Sociale, revue du CEDIAS - Musée social, n³ . Le dossier voit également les interventions 
elle invitait le secteur social à développer la pluridisciplinarité, à conventionner les centres de formation avec l'université (notamment via les licences en sciences de l'éducation et AES - Administration économique et sociale), dans un "esprit de décloisonne$m e n t{ }^{31}$. Le positionnement de cette ministre reste important pour l'époque également en raison de l'accent mis sur la " contractualisation " et l'élargissement du public de l'action sociale. Désormais, le travail social, entendu au sens de "moyen de contrôle et de transformation ", doit ouvrir ses compétences pour que tout public (et non seulement les " exclus") devienne un citoyen à part entière, sans perdre son pluralisme entre privé et public.

Dans le sillage des lois sur la décentralisation ${ }^{32}$, la création des IRTS (Instituts régionaux de travail social) en $1986^{33}$, centres de formation "multi-filière ", initie un mouvement censé développer la recherche et l'interdisciplinarité. Elle contribue au maintien de la formation sous une autorité " partagée », privée et publique (via les collectivités locales). Pourtant, alors que les IUT commencent à développer les diplômes qui deviendront les DUT "carrières sociales ", les rapports avec l'espace universitaire ne s'éclaircissent pas. Les formations professionnelles sont remises en cause, l'accent est mis sur la "mise au travail ", comme en atteste l'arrêté ministériel pris en juillet $1989^{34}$. Cet arrêté aligne le diplôme d'assistant-e social-e sur le Répertoire national des certifications professionnelles (RNCP) en lui reconnaissant un niveau III de qualification, équivalent au bac +2 . Dix ans après, ce sera la loi-cadre contre l'exclusion ${ }^{35}$ qui mettra l'accent sur le renforcement du lien de la formation des travailleurs sociaux avec les politiques sociales adoptées. Si la reconnaissance au niveau licence est revendiquée par la plupart des acteurs de cet espace, la volonté de rester hors de l'institution universitaire demeure forte, par crainte de dissoudre l'ensemble du travail social (en privilégiant un métier sur les autres) et par volonté de mettre en avant les "savoirs professionnels » et les "savoir-faire " acquis dans la pratique du métier.

Tout au long des années 1990, plusieurs rapports sont présentées (Molina, 2014, p. 135) en concertation entre les pouvoirs publics, les acteurs de la formation et des organismes privés associatifs, proposant différentes options, sans succès, alors même

d'autres acteurs de l'époque, comme Guy Raffi.

31. L'un des nouveaux leitmotiv des politiques publiques, notamment dans l'enseignement supérieur. Cf. «Le décloisonnement comme outil de changement ", Loi Fioraso 2013.

32. Loi du 22/07/1983, 83-663, et circulaire des 21/10/1983 et 4/11/1983, décret et circulaires du 19/10/1984 sur le transfert des services d'action sociale et santé aux conseils généraux, qui deviennent les principaux employeurs des ASS.

33. D'après l'idée développée par Bernard Lory (directeur général de l'Action sociale, de 1959 à 1966). Le premier institut nait bien avant, en 1975, sous le nom d'Institut régional de formation et de recherche en travail social, IRFRTS. René Lenoir en sera nommé premier directeur (Jaeger, 2012).

34. Arrêté du 26/07/1989.

35. Loi nº 98-657 du 29/071998 d'orientation relative à la lutte contre les exclusions. 
que ces formations restent éclatées et que le nombre de travailleurs sociaux s'accroît ${ }^{36}$. Simultanément, le développement de toute formation professionnelle dans l'université est établi en tant que priorité nationale ${ }^{37}$. Il aboutira à la création des licences professionnelles en 1999, puis à l'acceptation des priorités de Lisbonne en 2000. C'est notamment dans ce double processus - de "modernisation " de l'université et de mise à l'écart des formations professionnelles "traditionnelles »- que se situe la prolifération de formations universitaires d' "intervention sociale " (44 en 2008-2009, selon l'étude de Marc Fourdrignier, 2009).

Le contexte des années 2000 amène à une ultime reconfiguration de cet espace ${ }^{38}$. Les lois sur la décentralisation ${ }^{39}$ incluent cette formation dans les compétences transférées aux régions ; ces dernières assurent le financement des écoles en fonction du nombre de places " agréées » dont elles ont besoin au niveau local ${ }^{40}$, et représentent souvent les premiers employeurs des diplômés. L'arrêt du 29 juin 2004 se focalise sur ce diplôme et introduit des « référentiels » (professionnels, de compétence, de formation) qui atténuent les spécificités du métier. Modifié à nouveau par arrêt en 2011, cette réforme introduit le diplôme d'assistant-e social-e dans l'espace européen de l'enseignement supérieur : désormais, la formation est reconnue par 180 ECTS et se structure en "modules". Alors que la période de stage diminue progressivement au fil des années (12 mois au lieu de 14 mois depuis 1980), les centres de formation augmentent leur marge de manœuvre dans l'offre de formation (1 740 contre 1400 heures). Le poids des SHS (sciences humaines et sociales) diminue face à d'autres unités de formation, comme le droit ou la législation de politiques sociales. Enfin, des "disciplines " et "qualifications ", on passe aux " compétences » à valider au fil des trois ans.

On observe plus particulièrement ce processus de déspécialisation de la formation dans la réforme des épreuves du Diplôme d'État (DEASS) à valider au bout des trois ans de formation et qui marque l'entrée "officielle » dans le métier. Le mémoire "d'initiation à la recherche ", introduit lors de la réforme de 1980, et construit à l'image d'un mémoire universitaire inspiré des sciences humaines et sociales, est conservé. Une nouvelle épreuve voit également le jour : le «dossier de pratique professionnelle », dont la préparation commence dès la première année et qui certifie le premier « domaine de

36. Même la formation d'assistant social qui avait enregistré une baisse de recrutement, pendant les années 1980, accroît ses effectifs tout au long des années 1990 (Woitrain, 2000).

37. Notamment lors des Assises nationales de l'Enseignement supérieur de 1991.

38. La réforme de la VAE (Validation des Acquis de l'Expérience) de 2002 influence elle aussi tout particulièrement les formations en travail social, espace particulièrement investi par des personnes voulant reconvertir des expériences professionnelles dans le secteur social.

39. Loi no 2004-809 du 13 août 2004 relative aux libertés et responsabilités locales, et loi n ${ }^{\circ} 2014-288$ du 5 mars 2014 relative à la formation professionnelle, à l'emploi et à la démocratie sociale.

40. Dans le cas de l'Ile-de-France, les données de la DREES (Direction de la recherche, des études, de l'évaluation et des statistiques) affichent 548 étudiants en 2013. 
compétence » d'un assistant-e social-e. Ce dossier est composé d'une explicitation du positionnement professionnel de l'étudiant, qui doit analyser deux cas pratiques (l'un individuel et l'autre collectif) et produire une auto-évaluation de son parcours de formation, en parallèle d'un document d'évaluation produit par l'école. Ainsi, bien que la formation s'approche progressivement d'une uniformisation par rapport au modèle universitaire - les mois de stage diminuent, les heures théoriques augmentent, les crédits européens uniformisent - l'aspect professionnel, "pratique ", reste très présent dans son orientation.

Le cas du " mémoire " atteste de cette ambivalence. Dans l'une des écoles observées, c'est une formatrice psychologue qui s'occupe de l'organisation des mémoires, quand l'aspect pédagogique est délégué à des vacataires extérieurs. Le cas d'Océane ${ }^{41}$, étudiante en $3^{\mathrm{e}}$ année, est éclairant pour comprendre l'ambiguïté entre aspect théorique et professionnel. Sa tutrice est docteure en sociologie et collabore depuis peu avec son centre de formation. Présentant un mémoire sur l'application des contrats d'engagement réciproque dans le cadre du revenu de solidarité active, elle est confrontée à une remise en cause du projet porté dans son travail :

"Dans mon mémoire, j'écrivais que les ASS réclamaient la sanction et on [les formateurs, nda] m'a dit de nuancer parce ce n'est pas dans les valeurs de l'intervention sociale de sanctionner (...). Ma tutrice était une sociologue, pas une assistante sociale, elle m'a dit de rester sur la sanction; or, les formateurs m'ont dit de prendre de la distance par rapport à la "sanction" (...) Les formatrices me disaient: 'bah non, l'ASS n'est pas là pour sanctionner, elle est là pour appliquer un cadre règlementaire, elle a aucun pouvoir de sanctionner, donc il faut prendre de la distance par rapport à ce mot, ce qui n'est pas forcément un concept du travail social'. (...) Le jury blanc que j'avais eu trois semaines avant de rendre le mémoire était totalement fermé à la discussion, ils disaient que c'est pas intéressant d'aller, de travailler sur cet aspect, ils me disaient de défendre l'obligation de contractualisation, c'était pas dans la culture des ASS de sanctionner..."

$Q$ : Et tu en as parlé avec ta tutrice de mémoire?

"Je lui ai dit que dans mon mémoire, ils étaient contre cette notion, pour elle, ça ne posait pas problème, elle a demandé à d'autres sociologues qui lui disaient pas de problèmes, mais elle m'a dit de demander quand même aux formateurs, et après c'est moi qui ai fait le choix... C'était super difficile... Une fois au DE, on ne sera pas forcément face à un universitaire, mais à un praticien..."

Loin d'être un cas isolé, cet extrait témoigne de la dissonance, voire du conflit entre les aspects universitaire et professionnel. Même si le mémoire suit les méthodes des sciences sociales et une construction à vocation universitaire, il demeure un exercice

41. Discussion informelle avec Océane, 30 ans, père maçon, mère restauratrice, divorcés. Journal de terrain, avril 2016. 
évalué principalement par des professionnels, futurs pairs, lors du jury du diplôme d'État $^{42}$. Ce sont donc les " codes professionnels ", la logique de "service " aux individus, qui priment sur le "désintéressement " et l' "objectivation » de la recherche scientifique.

Contrairement au cas italien, qui suit un schéma plus « classique » de professionnalisation d'un groupe professionnel, le corps enseignant en travail social français contribue à se maintenir dans le flou. Le service est assuré par des formateurs aux profils divers dans chaque école, un diplôme du travail social et quelques années d'expérience dans les services ou - de plus en plus - des masters universitaires dans des disciplines de SHS, étant souvent suffisants à l'embauche. Parallèlement, les écoles s'appuient aussi sur des interve?nants extérieurs à l'école pour des cours tout au long du cycle d'étude : des professionnels ou des cadres des services, des doctorants ou docteurs sans poste s'ajoutent à quelques formateurs " titulaires " des écoles, eux-mêmes recrutés au sein de chaque école en fonction des profils et idéologies "spécifiques" (Verron, 2013). Pour ces vacataires aux profils forts hétérogènes, il n'y a pas consensus autour de l'identité ou du profil d'un " travailleur social ", ou encore d'un-e assistant-e social-e.

La formation en service social garde encore son ancrage régional et prend plusieurs formes : mono/multi-filières, généraliste ou spécialisée (hôpital, allocations familiales, collectivités territoriales, etc.), regroupant différents métiers du social (ASS, éducateurs spécialisés, éducateurs de jeunes enfants), avec un statut privé, public, universitaire (DUT), associatif, ou proposant un double cursus avec l'université (en sciences de l'éducation ou AES), ce qui peut entraîner les stratégies différenciés des étudiants qui s'orientent vers cet espace (Molina, 2015). Or, le récent développement de l'application "Admission Post-Bac" (APB) à la sortie du secondaire exclut les centres de formation régionaux, alors que la diffusion de "prépas aux concours sanitaires et sociaux » ancre ces filières dans des logiques de premier cycle de l'enseignement supérieur.

En dehors de la certification de qualifications professionnelles, le métier d'assistant-e social-e ne parvient pas à définir une " autonomie " propre et voit son " expertise " concurrencée tant par une université en reconfiguration, que par l'essor du travail associatif. Cette reproduction du corps professionnel, axée sur les praticiens du terrain, ne permet pas à cette filière de garder une "spécificité " face aux autres secteurs du travail social, et aux concurrences issues des nouvelles filières universitaires. Les discussions autour d'un " travailleur social unique " ou sur le rôle de " technicien du social " ou encore d'un "socle commun de compétences », introduites dans les derniers rapports ${ }^{43}$, ainsi que les difficultés grandissantes de plusieurs centres de formation dans

42. La commission est composée désormais de professionnels, de formateurs et de responsables des centres de formation, alors qu'il y a quelques années, des universitaires pouvaient y siéger.

43. Rapport "Reconnaître et valoriser le travail social » de Brigitte Bourguignon, députée PS, paru en septembre 2015, et rapport de la Commission professionnelle consultative du travail social et de l'intervention 
la recherche de stages pour leurs étudiants, attestent alors d'une reconfiguration du métier d'assistant-e social-e, en direction d'un risque de "déprofessionnalisation " de ce cursus. Pourtant, les nouvelles recompositions à l'œuvre dans l'université (pôles de recherche et d'enseignement supérieur - PRES, communautés d'universités et établissements - ComUE) qui voient déjà l'entrée des filières non-universitaires dans celle-ci, laissent présager des évolutions dans le rapport entre champ académique et espace du travail social.

\section{Conclusion : entre formation professionnelle et enseignement professionnalisé}

Les politiques de "modernisation» de l'enseignement supérieur, développées en Europe depuis vingt ans (Charle et Soulié, 2007), ont produit des situations différentes, dépendant souvent des spécificités des traditions nationales (Bourdieu, 2007, pp. 35-42). Elles ont rapproché l'université européenne d'inspiration humboldtienne ${ }^{44}$ de celle plus libérale d'inspiration anglo-saxonne. Il est intéressant d'observer alors que dans les revendications d'universitarisation du travail social, au fil du temps, c'est surtout l'inspiration anglo-saxonne qui primait.

Dans le cas italien, cette progressive universitarisation du service social s'opère au moment où la logique professionnelle s'introduit au sein de l'espace universitaire, où l'université " pénètre " dans le marché du travail (et vice-versa). Nous ne saurions réduire ces changements à une adaptation de ces écoles professionnelles au modèle académique (academic drift ou research drift), c'est l'institution universitaire même qui change ses perspectives. La stratégie poursuivie par les acteurs de ce champ organisationnel (Di Maggio et Powell, 1983) est celle, classique, des professions libérales. La question de la reproduction du corps professionnel se pose avec l'émergence d'un corps académique de "service social ». Simultanément, cela amène au développement progressif des filières professionnelles et à l'entrée de nouvelles étudiantes, auparavant exclues du jeu universitaire, dans un contexte de dévalorisation des titres universitaires et de diminution des effectifs étudiants.

Le cas français atteste davantage de ces ambivalences dans un pays où l'espace de l'enseignement supérieur est plus hétérogène. Ici, pour l'instant, un positionnement à l'écart du monde universitaire a été privilégié pour maintenir l'hétérogénéité professionnelle (le «travail social » au lieu de service social, éducation spécialisée...), alors que l'institution universitaire elle-même a progressivement changé de forme. L'espace

sociale (CPC) sur les formations sociales, à la Secrétaire d'État Ségolène Neuville, paru en octobre 2016. 44. Le modèle humboldtien (par le nom du ministre prussien de l'Education Von Humboldt) renvoie à la conception de l'université comme lieu de connaissance, qui réunit simultanément la fonction d'enseignement et la fonction de recherche (Charle et Soulié, 2007). 
pédagogique est occupé par des acteurs plus hétérogènes. Pour les écoles de travail social françaises, le rapprochement avec l'aspect académique recèle le risque de "tout perdre ", de ne plus avoir la main sur la maîtrise de la formation, ni sur les différents métiers qui s'entremêlent. La revendication de l'argument « à l'international » (l'existence de communautés académiques établies, de cursus en social work) est souvent portée comme la raison même de la création d'une discipline, d'un cursus ou d'un doctorat autonome ; néanmoins, nous avons vu comment chaque système national dépend aussi de logiques intrinsèques aux différents espaces de formation et combien il serait difficile de définir une formation homogène à un travail social " universel ».

Pourtant, que cela soit dans le cadre d'un schéma libéral protégé ou sous le contrôle de l'État, notre enquête atteste d'une distanciation progressive vis-à-vis du modèle d'assistant-e social-e estampillé "fonction publique " et caractérisé par l'attribution d'un service particulier. C'est alors un modèle de métier différent qui s'esquisse. Les changements des années 1990 (titre universitaire et ordre professionnel) et les évolutions récentes de la formation, en Italie, ou la concurrence des autres formations et métiers, en France, amplifient la dépendance du métier au marché de l'emploi et floutent les contours d'une identité professionnelle. L'espace du travail social composé par l'image classique de l'assistant-e social-e fonctionnaire se restructure, le travail social comme "service public » est remis en cause, qu'on y accède après une formation universitaire ou privée régionale. Le travail social développe des savoirs basés sur la centralité de la "pratique ", sur l'individualisation du parcours tant de l'étudiant que du service à l' " usager ", sur une culture du " projet professionnel ». Il demeure important de se demander aussi en quoi ces savoirs s'harmonisent avec le tournant actuel des politiques de l'enseignement supérieur à l'échelle européenne.

\section{Bibliographie}

Bernocchi R., Canevini Milena D., Cremoncini Vodia M., Ferrario F., Gazzaniga L., Dal Pra Ponticelli M. (1984), Le scuole di servizio sociale. Aspetti et momenti della loro storia, Fondazione E. Zancan, Padoue.

Bezes P. (2009), Les réformes de l'administration française (1962-2008), Presses Universitaires de France, Paris.

Bourdieu P. (2007), "Systèmes d'enseignement et systèmes de pensée ", in Deauvieau J. \& Terrail J.-P., Les sociologues, l'école et la transmission des savoirs, La Dispute, Paris, pp. $17-42$.

Charle C. \& Soulié C. (2007), Les ravages de la "modernisation " universitaire en Europe, Syllepse, Paris. 
Di Maggio P. \& Powell W. W. (1983), “The Iron cage revisited: institutional isomorphism and collective rationality in organizational fields", American Sociological Review, 48, pp. 147-160.

Fourdrignier M. (2009), "Professionnaliser les métiers du sanitaire et du social à l'université, une mission impossible ?", Formation Emploi, n 108, pp. 67-81.

Genieys W. (1999), "Sociologie des sommets de l'administration sanitaire et sociale 1981-1997 : des élites politico-administratives en question ? ", in Hassenteufel P. \& alii, L'émergence d'une "élite du welfare "? Sociologie des sommets de l'État en interaction Rapport de recherche, pp. 19-71.

Jaeger M. (2012), La coopération entre les établissements de formation préparant aux diplômes de travail social et les universités, Rapport commandité par la DGCS, 81 p.

Mastropaolo A. (2000), Antipolitica. All'origine della crisi italiana, La Nuova Italia, Firenze.

May P. \& Cacioppo M. (2015), "Il mercato del lavoro degli assistenti sociali ", in Tognetti Bordogna M., Voglio fare l'assistente sociale, Roma, Franco Angeli, pp. 67-94.

Molina Y. (2014), Les travailleurs sociaux, des groupes professionnels en transformation, Thèse de doctorat en sociologie, EHESS, Paris.

Molina Y. (2015), «L'accès aux formations sociales, entre choix d'orientation professionnelle et stratégies ", Formation Emploi, n 132, pp. 117-137.

Morand-Durocher G. (1992), Identité professionnelle et formation permanente des assistantes sociales, Bayard, Paris.

Neave G. (1979), "Academic drift: Some views from Europe”, Studies in Higher Education, 4(2), pp. 143-159.

Pitzalis M. (2007), «L'université italienne entre marché, formations professionnelles et pouvoir politique ", in Charle C. \& Soulié C., Les ravages de la "modernisation" universitaire en Europe, Syllepse, Paris, pp. 69-88.

Rater-Garcette C. (1996), La professionnalisation du travail social, L'Harmattan, Paris.

Rullac S. (2014), La scientifisation du travail social, Presses de l'EHESP, Rennes.

Tanguy L. (2005), "De l'éducation à la formation : quelles réformes ? », Education et sociétés, $\mathrm{n}^{\circ} 16$, pp. 99-122.

Tognetti Bordogna M. (2015), Voglio fare l'assistente sociale, Roma, Franco Angeli.

Verron C. (2013), Les formateurs en travail social: une professionnalisation impossible, Thèse de doctorat en sociologie, Université de Bretagne occidentale. 
Viguier F. (2010), La cause des pauvres. Mobilisation humanitaire et transformations de l'Etat social en France (1945-2010), Thèse de doctorat en sociologie, EHESS.

Woitrain E. (2000), « Les travailleurs sociaux en 1998, environ 800000 professionnels reconnus ", Etudes et résultats, ${ }^{\circ} 79$.

Woolf S. (2003), «A propos de la réforme de l'Université italienne : rapports de force et paradoxes structurels " in Schultheis F., Roca I., Escoda M., Cousin P.-F., Le cauchemar de Humboldt. Les réformes de l'enseignement supérieur européen, Raisons d'agir, Paris. 